\title{
La Medicina Legal en su momento más decisivo. Exigencias formativas de los especialistas en Medicina Legal y Forense, y de los Médicos Forenses
}

No vamos a volver al Once upon a time... para rememorar las situaciones pasadas en las que fue posible el necesario encuentro entre la Medicina Legal y la Medicina Forense en España. Considero cierto que el encuentro entre los Departamentos de Medicina Legal y los especialistas que trabajábamos en ellos, y los Médicos Forenses, primero adscritos a sus juzgados correspondientes y después agrupados en los Institutos de Medicina Legal, habría tenido todas las ventajas de una Medicina Legal y Forense moderna, eficaz y eficiente en sus fines periciales, de docencia y de investigación; sólo había que mirar la organización que esta disciplina tenía en el vecino país de Portugal y en la mayoría de los países de Europa, y hacer algo semejante.

La Ley Orgánica del Poder Judicial fue fallida en este aspecto esencial para la Administración de Justicia. En las normas derivadas de ella con la creación de los Institutos de Medicina Legal se estableció un débil puente, a través de las Comisiones de Docencia de los mismos, con la presencia de un profesor de Medicina Legal, pero sólo contemplaban aspectos formativos y de investigación. Sin embargo, no se entró en la auténtica reforma de la integración de ambas estructuras, la dependiente del Ministerio de Justicia organizada para el abastecimiento pericial de los jueces y tribunales, y la dependiente del Ministerio de Educación dirigida a la investigación y a la docencia, fundamentalmente para la formación medicolegal de los futuros médicos y de los especialistas en Medicina Legal y Forense dirigidos a la pericia privada.

La posibilidad de acceder, por oposición, al Cuerpo de Médicos Forenses sin tener el título de especialista en Medicina Legal y Forense, y de otro lado el hecho de que tener el título de especialista sólo abriera la puerta al ejercicio pericial privado, no favorecían esta unificación deseada y necesaria.

Sin embargo, estoy segura de que esto va a ser historia, porque hoy más que nunca nos enfrentamos al ser o no ser de la Medicina Legal y Forense como especialidad médica. La convocatoria MIR de 2015 será la última en la que se convoquen plazas de especialistas (en régimen de Escuela profesional), ya que sólo se reconocerán las especialidades médicas que se cursen dentro del modelo de troncalidad y con formación hospitalaria.

Tenemos un año para convencer a los Ministerios de Justicia y de Sanidad de que eso es posible para la Medicina Legal y Forense con los recursos de que hoy disponemos, unos propios del Ministerio de Justicia (Institutos de Medicina Legal, Instituto Nacional de Toxicología y Ciencias Forenses...) y otros propios de los departamentos universitarios de Medicina Legal.

En mi opinión, en este momento todos los que nos dedicamos a esta Medicina estamos de acuerdo en que las circunstancias científicas y sociales la hacen más necesaria que nunca como una especialidad reconocida y con especialistas formados acorde con las exigencias de rigor que hoy demanda la sociedad en todos los ámbitos médicos.

$Y$ es en este aspecto en el que me centraré a partir de ahora, en la necesidad de una formación para los Médicos Forenses acorde con las necesidades de una prueba pericial rigurosa y sólida, y basada en los conocimientos que hoy aportan a la Medicina Legal las parcelas de la Medicina de que ésta se nutre y a las que recurre, atendiendo a la naturaleza del caso que se juzga y a las cuestiones médicas que éste plantea.

El núcleo duro de la Medicina Legal y Forense siempre fue la Patología; los mecanismos de muerte y las lesiones de naturaleza violenta estaban en la base de la mayoría de las pericias médico-forenses. Sin embargo, la "medicina de los muertos" se ha ido haciendo cada vez más una "medicina de los vivos" que reclaman justicia por los daños sufridos.

La evidencia del grave problema de la violencia familiar o doméstica, y en concreto la violencia contra las mujeres (violencia de género), ha supuesto una exigencia formativa de primer orden para los Médicos

\section{Castellano Arroyo}

Catedrática de Medicina Legal y Forense.

Académica de la Real Academia de Medicina de Andalucía oriental y de la Real Academia Nacional de Medicina

Correspondencia:

M. Castellano Arroyo Departamento de Medicina Legal. Facultad de Medicina. Unversidad de Granada. Avda. de Madrid, 11. 18071 Granada.

E-mail: mcarroyo@ugr.es 
Forenses. Se produjo la respuesta legal y social, con cambios sucesivos que agravaron las penas y con medidas de protección nunca antes conocidas para las víctimas de estos delitos. Sin embargo, año tras año y a pesar de los medios y recursos de que se dispone, las agresiones se suceden y las muertes se mantienen o incluso aumentan.

Todo ello guarda estrecha relación con el papel de la Medicina Legal y Forense en este y otros problemas.

Decía antes que el objeto princeps de la Medicina Legal había sido la Patología forense, realizar bien la autopsia; interrogar al cadáver desde la bioquímica, la anatomía patológica, la toxicología, la antropología y hasta la genética ha sido preocupación para los Médicos Forenses que han organizado y realizado numerosos cursos de actualización alrededor de estos temas, e incluso programas de especialización en Servicios de Anatomía Patológica. Estamos de acuerdo en esto, porque sólo una autopsia bien hecha podrá dar todas las claves y respuestas para resolver con acierto un caso. $Y$ hay que reconocer que el objetivo se va cumpliendo de forma adecuada con escasas excepciones.

Sin embargo, otras parcelas médico-forenses no han recibido la misma atención formativa, y nuestra Medicina Forense presenta algunas deficiencias que, si no se remedian, la irán relegando frente al imparable avance de la mayoría de las especialidades médicas cabalgando sobre las nuevas tecnologías aplicadas al diagnóstico y a la terapéutica, y en las que incluso van apareciendo áreas de superespecialización.

La adecuada preparación médico-forense necesita un programa formativo que incluya los conocimientos teóricos propios de nuestra especialidad. El extenso programa de las oposiciones de Médicos Forenses contempla estos contenidos teóricos, pero lo criticable es que esta formación teórica no lleva paralela la correspondiente formación práctica que prepare y haga competente al Médico Forense para realizar por sí mismo los actos médicos propios de su función. La Medicina al servicio de la Administración de Justicia. ¿Cómo puede pensarse que ese auxilio a la Administración de Justicia pueda hacerse en el siglo XXI por un médico no especializado, o por un médico de una especialidad concreta, formado para el diagnóstico y el tratamiento, pero sin formación en el lenguaje medicolegal, la organización judicial, los objetivos del derecho civil o penal, o la regulación de la prueba pericial médica?

Una formación y una capacitación medicolegal no se improvisan, requieren saber medicina, pero con la perspectiva de saberla aplicar a las necesidades de los jueces y tribunales en los problemas concretos que estudian, analizan y sentencian en las diferentes jurisdicciones.

Es difícil reconocer y elegir cuáles son los conocimientos médicos generales, o los de una especialidad médica, que son necesarios para cumplir los fines de la Medicina Legal y Forense en cada caso concreto, el Ilamado "objeto de la pericia". En esto reside la mayor dificultad de nuestra misión médico-forense, en realizar y proporcionar un informe que dé una respuesta necesaria y suficiente para que el juez resuelva eficazmente y con justicia el caso; sólo así la pericia médica habrá cumplido su función. No basta con emitir el informe si éste no llega al fondo del asunto, si no ofrece la información más completa posible.

Por ello era necesario abandonar el modelo del Médico Forense pluripotencial capaz de saberlo todo de todo. La agrupación de los Médicos Forenses en los Institutos de Medicina Legal permite la adscripción a un Servicio concreto: Patología, Clínica médicoforense, Laboratorio, etc., lo que permite centrarse en una tarea específica y, aún con más trascendencia, compartir, consultar y debatir dudas con los compañeros, que siempre aporta más información y rigor al informe.

Sin embargo, hay algunas parcelas de conocimiento médico-forense que se han mantenido, entre los Médicos Forenses, envueltas en una cierta penumbra, y me refiero a una muy querida para mí y a la que me dedico con bastante asiduidad: la Psiquiatría Forense o Medicina Legal Psiquiátrica.

La historia nos dice que la Psiquiatría clínica fue una de las últimas especialidades clásicas en tener autonomía, desgajándose ésta de la Medicina Legal. Yo aún tuve la suerte de conocer a Don Ramón Rey Ardid, primer catedrático de Psiquiatría de Zaragoza (además de campeón de España de ajedrez), que hasta mediada la década de 1960 había sido Profesor Adjunto de Medicina Legal junto a Don Valentín Pérez Argilés, catedrático de la asignatura (ambos eran psiquiatras y estaban considerados como las dos mentes más clarividentes y privilegiadas del Aragón de la época).

La Psiquiatría en su origen fue estudio y sistematización de los síntomas y signos para conocer la enfermedad, pero los tratamientos eficaces estaban aún por descubrir. Estas circunstancias explican que la mayor atención que recibían estos enfermos venía desde el Derecho y en relación con conductas antijurídicas que era necesario valorar para establecer la imputabilidad de la persona que las había realizado. Era necesario escrutar la mente de la persona enfer- 
ma para conocer hasta dónde sus actos materiales estaban guiados por la razón y por la voluntad o capacidad de elegir. Igualmente era motivo de pericia medicolegal establecer la capacidad civil de las personas con enfermedad mental, para decidir sobre la protección de la propia persona y de sus bienes, bajo la tutela judicial.

Estas pruebas periciales no estaban exentas de dificultad, pero se buscaban síntomas y signos graves y evidentes que podían detectarse desde unos conocimientos psiquiátricos básicos y propios del programa aprendido por los Médicos Forenses.

Los requerimientos actuales de la Psiquiatría Forense o Medicina Legal psiquiátrica son mucho más extensos, y por ello es un desafío para el Médico Forense que no puede renunciar a formarse y adquirir la capacitación necesaria para abordar una pericia médico-psicológica psiquiátrica con plena autonomía.

La Psicología médica forma parte de la formación general del médico. Conocer las funciones mentales y su integración extraordinaria en la "unidad psicofísica" que es la persona humana, en la que todo lo orgánico se experimenta psíquicamente y todo lo psíquico se expresa orgánicamente, es un desafío para el Médico General y para el Médico Forense en particular, ya que en la base de las actuaciones forenses suele existir un acontecimiento vital estresante y con capacidad para desestabilizar e influir en la salud psíquica de las personas.

El Médico Forense que tiene encomendada la clínica médico-forense se va a enfrentar a la exploración y la valoración de personas que vienen por los más diversos motivos: una mujer víctima de una agresión sexual, de violencia física o psíquica continuada; una persona víctima de una agresión, de un accidente de tráfico o de trabajo; los autores de un homicidio o de una agresión; la víctima de una presunta mala praxis médica, etc. En algunos de estos casos habrá que valorar de forma expresa el daño psíquico como secuela, y junto a todo esto, la persona sobre la que hay que determinar su imputabilidad o su capacidad civil.

La exploración de la que se derive un informe pericial adecuado y acorde con las características de cada caso necesita un Médico Forense con una formación que abarque el conocimiento de las lesiones físicas, de sus necesidades terapéuticas y de las secuelas a que éstas dan lugar; sin embargo, junto a ello, el Médico Forense también debe estar preparado para realizar una exploración psicológico-psiquiátrica básica que le permita conocer la personalidad del lesionado, de la víctima o del agresor a quien entrevista y valora. Detrás de cada caso médico-forense hay una persona, con su manera de ser, de sentir, de percibir el entorno, de elaborarlo y de responder a éste con una conducta que es propia y personal, y que desde luego es previsible, y evitable, cuando el caso así lo aconseje.

Esta preparación práctica en una exploración complementaria médico-psiquiátrica no es difícil para el actual Médico Forense, a quien ya se le ha exigido para serlo que conozca la teoría de la exploración psicométrica y psicodiagnóstica a través de todos los tests, cuestionarios y escalas conocidos en la práctica habitual. Estamos seguros de que en un futuro próximo la especialidad de Medicina Legal y Forense será requisito obligado para ser Médico Forense, en cuyo caso será el programa de la especialidad el que contenga la necesaria formación teórico-práctica en Psiquiatría Forense.

En este momento, atendiendo a lo dicho, y siguiendo con los actuales Médicos Forenses, se trataría de complementar esos conocimientos teóricos con una capacitación en la administración y la interpretación de una batería de tests sencilla, que proporcione al Médico Forense información sobre la personalidad normal o patológica de la persona que explora, para detectar trastornos de personalidad y otros trastornos mentales de mayor gravedad, y datos muy importantes, como son la presencia y el grado de ansiedad, o la depresión, que tanto condicionan las conductas humanas hacia la autolesión o las agresiones a los demás.

Como hemos dicho, este complemento formativo es fácil de adquirir sobre los conocimientos ya demostrados en la oposición, y constituiría para el Médico Forense un instrumento sensacional para fundamentar muchos informes en los que objetivar la sinceridad de lo manifestado por la persona explorada, o la realidad de una secuela que afecta a la salud psíquica, o el riesgo que tiene la persona explorada de llevar a cabo conductas dañinas hacia otras personas, y tantas y tantas otras cosas que surgen de los resultados de las pruebas psicodiagnósticas.

Sabemos que afortunadamente los Institutos de Medicina Legal, y los Juzgados de Violencia de Género en particular, disponen de psicólogos, profesionales expresamente formados para la exploración psicológica. Esta estructura de apoyo es muy importante para la calidad pericial e imprescindible para numerosas pericias psiquiátricas especialmente complejas, por lo que requieren pruebas más numerosas y especializadas. Sin embargo, si toda la exploración médicoforense en el ámbito de lo psiquiátrico-psicológico recae en el Servicio de Psicología, se corre el riesgo de que las exploraciones sufran un importante retraso 
para la evacuación del informe pericial, hasta el punto de que en muchos casos el Médico Forense no pueda esperar al resultado de la exploración psicológica y opte por un informe limitado a la detección de síntomas graves (orientación, alucinaciones, ilusiones, trastorno grave del estado de ánimo...), dejando sin evaluar otros síntomas de gran importancia en el estudio completo del caso y, como exige una justicia de calidad, sirviéndose de los instrumentos objetivos adecuados que generen un informe útil, acorde con la realidad del caso y próximo a lo más justo.

Si queremos una justicia de calidad necesitamos un informe o pruebas periciales de calidad, y eso significa buenos peritos. Por ello, para la pericia médico-forense de calidad, el Médico Forense debe hacer el esfuerzo de adquirir una capacitación básica en Psicología-Psiquiatría Forense.

Éste es un reto que tienen planteado actualmente los Médicos Forenses, y que tendrán con mayor motivo en el afortunado caso de que la especialidad de Medicina Legal quede recogida como una especialidad médica similar a las ya comprendidas en el Tronco Médico, en el Borrador de Anteproyecto de Especialidades Médicas.
Termino reiterando el deseo de colaboración de todos los que hemos comprometido nuestra vida académica y profesional en el reconocimiento y el progreso de la Medicina Legal y Forense como una especialidad médica. En el momento actual, y en el futuro, la especialización necesitará un programa teórico (quizá a través de un Curso Superior de Medicina Legal) y un programa práctico que capacite a los especialistas en todas las áreas de la Medicina Legal, desde luego la Patología forense, pero también la Psiquiatría forense, la Sexología forense, la Medicina forense de la familia y del trabajo, y todas las áreas de estudios complementarios (toxicología, anatomía patológica, antropología, etc.) y básicos que se consideren.

Somos muchos los que, desde una larga experiencia, queremos que la Medicina Legal y Forense sea una especialidad médica valorada, admiramos y queremos a los Médicos Forense, y pensamos que el Ministerio de Justicia puede seguir seleccionándolos de entre los especialistas, y desde luego estamos dispuestos a colaborar para que sea posible un futuro de mayor esplendor y reconocimiento para la prueba pericial médico-forense.

\section{Nota}

Este artículo lo terminamos el día 3 de abril de 2014. Hoy, 5 de abril, justo antes de enviarlo, hemos conocido que en el Consejo de Ministros de ayer viernes se aprobó el anteproyecto de Ley Orgánica del Poder Judicial, que derogaría a la actualmente vigente. El anteproyecto recoge que en el futuro, para ser Médico Forense, será requisito imprescindible tener el título de Especialista, y sería lógico que lo fuera en Medicina Legal y Forense. Esto cumple las expectativas de los profesores de Medicina Legal y de muchos Médicos Forenses que, a lo largo de nuestra vida académica y profesional, hemos trabajado para que esto se hiciera realidad. Ahora estamos seguros de que la especialidad puede estar garantizada, y sólo nos queda esperar que se organice lo mejor posible y que en las correspondientes Unidades Docentes se aprovechen todos los recursos disponibles, humanos e instrumentales, tanto los del Ministerio de Justicia (Institutos de Medicina Legal, Instituto Nacional de Toxicología y Ciencias Forenses, y otros) como los propios del Ministerio de Educación ubicados en los Departamentos de Medicina Legal. 